\title{
Numerical Prediction of Hydrodynamic Loading on Circular Cylinder Array in Oscillatory Flow Using Direct-Forcing Immersed Boundary Method
}

\author{
Ming-Jyh Chern, ${ }^{1,2}$ Wei-Cheng Hsu, ${ }^{1}$ and Tzyy-Leng Horng ${ }^{3}$ \\ ${ }^{1}$ Department of Mechanical Engineering, National Taiwan University of Science and Technology, \\ Taipei 10607, Taiwan \\ ${ }^{2}$ Ecological Engineering Research Center, National Taiwan University, Taipei 10617, Taiwan \\ ${ }^{3}$ Department of Applied Mathematics, Feng Chia University, Taichung 40724, Taiwan \\ Correspondence should be addressed to Ming-Jyh Chern, mjchern@mail.ntust.edu.tw
}

Received 20 January 2012; Revised 28 June 2012; Accepted 20 July 2012

Academic Editor: Carl M. Larsen

Copyright (c) 2012 Ming-Jyh Chern et al. This is an open access article distributed under the Creative Commons Attribution License, which permits unrestricted use, distribution, and reproduction in any medium, provided the original work is properly cited.

\begin{abstract}
Cylindrical structures are commonly used in offshore engineering, for example, a tensionleg platform (TLP). Prediction of hydrodynamic loadings on those cylindrical structures is one of important issues in design of those marine structures. This study aims to provide a numerical model to simulate fluid-structure interaction around the cylindrical structures and to estimate those loadings using the direct-forcing immersed boundary method. Oscillatory flows are considered to simulate the flow caused by progressive waves in shallow water. Virtual forces due to the existence of those cylindrical structures are distributed in the fluid domain in the established immersed boundary model. As a results, influence of the marine structure on the fluid flow is included in the model. Furthermore, hydrodynamic loadings exerted on the marine structure are determined by the integral of virtual forces according to Newton's third law. A square array of four cylinders is considered as the marine structure in this study. Time histories of inline and lift coefficients are provided in the numerical study. The proposed approach can be useful for scientists and engineers who would like to understand the interaction of the oscillatory flow with the cylinder array or to estimate hydrodynamic loading on the array of cylinders.
\end{abstract}

\section{Introduction}

Marine structures are commonly utilized to undertake activities in the offshore region. For example, to obtain oil in the offshore region, a tension-leg platform (TLP) is used. Another example is the wave power station proposed by a Norwegian company [1]. The TLP frame is used for the wave power station. A platform with four circular cylinders is used as 
the main frame of the proposed wave power station. Since those marine structures are in the offshore region, hydrodynamic loading from waves and currents should be considered while one designs this sort of marine structures. Circular cylinders are often utilized as the frame of the marine structure, so prediction of the loadings on the circular cylinders is important. The aim of this study is to establish a numerical model to predict hydrodynamic loading exerted on those circular cylinders in marine structures.

Oscillatory flows are regarded as the flows due to a progressive wave train in shallow water, so interaction of oscillatory flows with cylinders have been studied by several researchers. Sumer and Fredsoe [2] reviewed published papers regarding studies of flow around a single cylinder in oscillatory flows. Keulegan-Carpenter (KC) number was used to categorize the flow variations into a variety of flow regimes. The study of hydrodynamic loadings exerted on a single circular cylinder in oscillatory flows can be found in numerous publications. Williamson [3] conducted a series of experiments to measure inline force and lift of an oscillating cylinder. Flows were visualized at various KC numbers. Sarpkaya [4] provided theoretical and experimental results of inertia coefficient and flow visualization. Obasaju et al. [5] measured forces on a circular cylinder in oscillating flow. They categorized the flow variations into asymmetric, the transverse, the diagonal, the third vortex, and the quasi-steady regimes as KC varied from 4 to 55.

Numerical studies on the force prediction for a single cylinder in oscillatory flows have been reported in the past two decades. Lin et al. [6] employed a hybrid Lagrangian/Eulerian discrete vortex method to simulate flow around a circular cylinder in oscillatory flow up to KC numbers of 30. Iliadis and Anagnostopoulos [7] considered viscous oscillatory flows interacting with a circular cylinder by solving streamfunction and vorticity formulation of the Navier-Stokes equation. The finite element method was adopted in their study. They visualized flow variations using vorticity contours at various KC numbers. The inline and the transverse forces on the circular cylinder were determined and reported. Zheng and Dalton [8] utilized the finite difference method to study the interaction between a rectangular cylinder and oscillatory flows. Variations of flow due to the change of $\mathrm{KC}$ number were visualized in their study. Prediction of forces on the square cylinder was also predicted in their model. An et al. [9] investigated the oscillatory flow around a circular cylinder at low KC numbers. Honji vortices were simulated and explained in their study.

Interaction of oscillatory flows with a number of cylinders has been reported in several papers. Williamson [3] studied flow around a pair of circular cylinders in his flow visualization experiments. Those two cylinder were either side-by-side or at $45^{\circ}$. The effect of gap between two cylinders on flow variation and forces were reported. Chern et al. [10] simulated oscillatory flows around a pair of side-by-side cylinders up to KC of 15 . Phase diagrams of force components were revealed to observe the route to chaos in the flow. The relationship between two vortical systems behind cylinders were analyzed. Anagnostopoulos and Dikarou [11] established a numerical model to simulate viscous oscillatory flow past four cylinders. The cylinders were in a square arrangement. Inline and transverse forces of the cylinders were predicted and the relationship among them and $\mathrm{KC}$ was given in the study. Also, the effect of pitch ratio on the viscous oscillatory flows was discussed.

An immersed boundary method which added a virtual force in the momentum equations to simulate the existence of solid has been receiving more and more attentions. One of immersed boundary methods is the so-called direct forcing method proposed by Yusof [12]. The direct forcing method determine a forcing term by calculating the difference between the interpolated velocities on the boundary points and the desired solid boundary velocities. As a result, it does not require to fit grids for a complex configuration. A Cartesian 
grid can be used in the direct-forcing immersed boundary method. The principle of the direct forcing method has been adopted and applied to several fluid-structure interactions in Fadlun et al. [13], Tseng and Ferziger [14], and Verzicco et al. [15]. The present model based on the direct forcing method was also validated by the uniform flow past cylinders in the work by Noor et al. [16]. In terms of those studies, it turns out that the direct-forcing immersed boundary method is able to simulate fluid-solid interactions and to predict hydrodynamic loadings on cylinders properly.

The aim of this study is to utilize the direct-forcing immersed boundary method to study the oscillatory flow around a cylinder array in a square arrangement. Since the geometric configuration of the cylinder array is complex in the flow domain, one can take the advantage of the propose direct-forcing immersed boundary method to simulate the oscillating flow with the complex cylinder configuration in Cartesian grids. The other advantage of the direct-forcing immersed boundary method is the prediction of forces on a solid immersed in fluids. The virtual force in the model can be used to determine forces. It is common that forces on a single cylinder in an oscillatory flow are divided to two parts, the drag and inertia forces (see Williamson [3] and Sarpkaya [4]). Nevertheless, the resultant forces of the cylinder in this study can be calculated by the integral of the virtual force in the solid region. Consequently, the oscillatory flow interacting with the cylinder array and the hydrodynamic loadings on the cylinder array can be obtained using the proposed model.

\section{Mathematical Formulae and Numerical Model}

In the present work, the direct forcing method is used to establish the proposed immersed boundary method. A virtual force is added to the incompressible Navier-Stokes equations in order to accommodate the interaction between solids and fluids. A solid body is identified by a volume-of-body function $\eta$ which denotes a fraction of solid within a cell. For a cell full of solids, $\eta$ is equal to 1 while it becomes 0 for a cell full of fluids. It is fractional in a boundary cell which consists of solids and fluids both.

\subsection{Equations of Fluid Motion}

An incompressible fluid is considered in the study. The motion of fluids should satisfy the conservation laws of mass and momentum. Mathematically, those physical laws can be denoted in dimensionless form and shown as

$$
\begin{gathered}
\nabla \cdot \mathbf{u}=0, \\
\frac{\partial \mathbf{u}}{\partial t}+\nabla \cdot(\mathbf{u u})=-\nabla p+\frac{1}{\operatorname{Re}} \nabla^{2} \mathbf{u}+\eta \mathbf{f},
\end{gathered}
$$

where $\mathbf{u}$ and $p$ are dimensionless velocity and pressure, respectively. Equations (2.1) and (2.2) are nondimensionalized by the characteristic velocity $U_{m}$ which is the amplitude of the oscillating velocity and the characteristic length $D$ which is the diameter of a circular cylinder. Meanwhile, time is nondimensionalized by the characteristic time $D / U_{m}$. Re is Reynolds number and defined by $U_{m} D / v$, where $v$ is kinematic viscosity. The proposed immersed boundary model adds the virtual force $\mathbf{f}$ to include the effect of solid in the viscous flow. 
The momentum equation (2.2) is solved by three steps. First, the velocity is stepped from the $m$ th time level to the first intermediate level $\mathbf{u}^{*}$ by solving (2.2) without the pressure gradient and virtual force at the beginning of each time step. This step is implemented by the following formula:

$$
\frac{\mathbf{u}^{*}-\mathbf{u}^{m}}{\Delta t}=S^{m}
$$

where $S$ includes the convective and diffusive terms of (2.2). Apparently, the first intermediate velocity $\mathbf{u}^{*}$ in (2.3) does not satisfy (2.1), so $\mathbf{u}^{*}$ is marched to the second intermediate velocity $\mathbf{u}^{* *}$ by including the pressure gradient,

$$
\frac{\mathbf{u}^{* *}-\mathbf{u}^{*}}{\Delta t}=-\nabla p^{m+1 / 2}
$$

at the second step. Taking the divergence of (2.4) gives

$$
\frac{\nabla \cdot \mathbf{u}^{* *}-\nabla \cdot \mathbf{u}^{*}}{\Delta t}=-\nabla^{2} p^{m+1 / 2}
$$

In principle, the second intermediate velocity $\mathbf{u}^{* *}$ should satisfy the continuity equation, that is,

$$
\nabla \cdot \mathbf{u}^{* *}=0
$$

Substitution of (2.6) to (2.5) results in the Poisson equation of pressure:

$$
\nabla^{2} p^{m+1 / 2}=\frac{1}{\Delta t} \nabla \cdot \mathbf{u}^{*}
$$

Once (2.7) is solved, $\mathbf{u}^{* *}$ will be obtained by (2.4). So far, the effect of solid on the viscous fluid flow has not been included. The virtual force $f$ caused by a solid should be involved at the third step, so $\mathbf{u}^{* *}$ is updated to the $(m+1)$ th time level by imposing the virtual force term, that is,

$$
\frac{\mathbf{u}^{m+1}-\mathbf{u}^{* *}}{\Delta t}=\eta \mathbf{f}^{m+1}
$$

The dimensionless virtual force $\mathbf{f}$ reveals the existence of a force to hold or to drive a solid body which is either stationary or moving. Given that the velocity of a solid is $\mathbf{u}_{s}$ and may be not the same as the calculated velocity $\mathbf{u}^{* *}, \mathbf{f}$ acting on the solid will have to ensure that the fluid velocity $\mathbf{u}^{m+1}$ is equal to the solid velocity $\mathbf{u}_{s}$ at the $(m+1)$ th time step, that is, $\mathbf{u}^{m+1}=\mathbf{u}_{s}^{m+1}$. Hence, the virtual force is defined as the rate of momentum change of a solid body and proportional to the difference between the solid velocity at the $(m+1)$ th time step 
and the fluid velocity at the $m$ th time step. The force exists at the fluid domain, where the solid body is immersed and zero elsewhere. Furthermore, it can be simply written as

$$
\mathbf{f}^{m+1}=\frac{\mathbf{u}^{m+1}-\mathbf{u}^{* *}}{\Delta t}=\frac{\mathbf{u}_{s}^{m+1}-\mathbf{u}^{* *}}{\Delta t}
$$

Presumably, cylinders are fixed so $\mathbf{u}_{s}$ is always zero for all cylinders.

\subsection{Oscillatory Flow Boundary Condition}

Oscillatory flows are considered in this study. Transient velocity boundary conditions are imposed at four boundaries of the computational domain to simulate oscillatory flows. Consider an oscillatory flow of dimensionless period $T$. The dimensionless horizontal velocity component of the oscillatory flow varies according to the condition

$$
u=\sin \left(\frac{2 \pi t}{T}\right)
$$

This boundary condition has been used by Zheng and Dalton [8] and Chern et al. [10] for simulations of oscillatory flows with square cylinders. The dimensionless vertical velocity component of the oscillatory flow at four boundaries of the computational domain is set as zero during simulations.

\subsection{Calculation of Hydrodynamic Force on Cylinder}

The integration of the virtual force will be a good approximation of the resultant dimensionless force exerted on a single circular cylinder,

$$
\mathbf{F}=-\iint_{\Omega} \eta \mathbf{f} d A
$$

where $\mathbf{F}$ is the resultant hydrodynamic force vector and $A$ is the area occupied by the circular cylinder. The inline force coefficient $C_{f}$ in the oscillatory flow direction can be denoted as

$$
C_{f}=-2 F_{x}
$$

and the life or transverse force coefficient $C_{l}$ can be determined by

$$
C_{l}=-2 F_{y}
$$

\subsection{Numerical Procedure to Predict Fluid-Solid Interaction}

The numerical procedure to predict viscous oscillatory flows and to trace the falling object at each time step can be summarized in the following steps. 


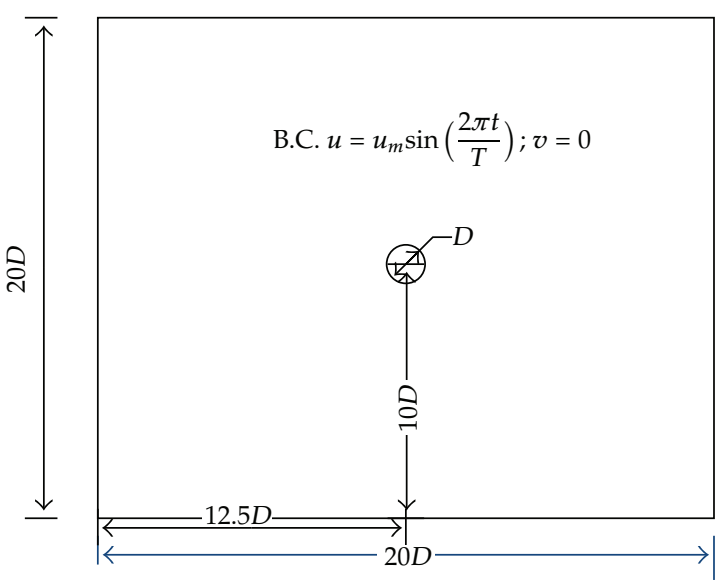

Figure 1: Schematic of interaction of an oscillatory flow with a single circular cylinder.

(1) Determine $\eta$ through the positions and diameters of cylinders at the first time step.

(2) Calculate $\mathbf{u}^{*}$ via (2.3).

(3) Solve the pressure Poisson equation (2.7) and advance to $\mathbf{u}^{* *}$ via (2.4).

(4) Compute the virtual force required via (2.9).

(5) Determine the velocity via (2.8) and back to Step 2 and for the next time step.

Uniform Cartesian grids are adopted in this study. $250 \times 250$ and $430 \times 430$ grids are used for cases for a single cylinder and four cylinders, respectively. The finite volume method is used to solve the momentum equations. The advective scheme is discretized by the so-called third QUICK scheme. The Adams-Bashforth scheme is used for the temporal derivative. The dimensionless time increment $\Delta t$ is set as $10^{-4}$ which satisfies the CFL condition so the stability can be retained in the model. The total dimensionless time for simulations is 230. It takes about 2 and half days for each simulation of the 2-D oscillatory flow around a single cylinder at a PC cluster consisting of Intel Xeon CPUs.

\section{Results and Discussion}

\subsection{Validation of Numerical Model}

Although the proposed numerical immersed boundary model was validated in our previous study (Noor et al. [16]) which considered a uniform past circular cylinders, it will be validated by the study of the interaction of oscillatory flow with a single circular cylinder again. Figure 1 shows the schematic of the benchmark test problem. Consider a circular cylinder of diameter $D$ in a fluid domain. The oscillatory flow is featured by KeuleganCarpenter $(\mathrm{KC})$ number which is determined by the formula $U_{m} T / D$. Cases at $K C=2$ and 10 and $\operatorname{Re}=200$ are simulated in the validation test. Figure 2 presents the evolution of vorticity contours during a cycle at $K C=2$. It is found that a pair of symmetric vortices appearing in two sides of the cylinder alternatively due to the change of flow direction. The results agree with Iliadis and Anagnostopoulos' study [7]. Furthermore, the development of the symmetric wake in half a cycle is calculated and shown in Figure 3. The wake is 

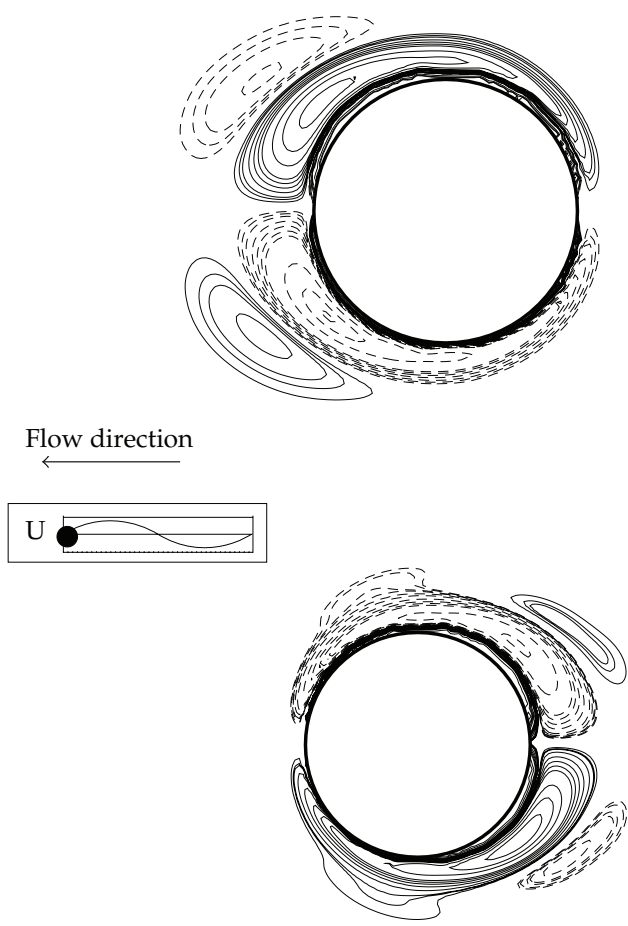

Flow direction

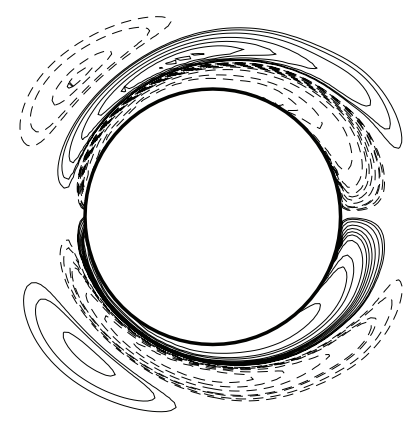

Flow direction

Flow direction
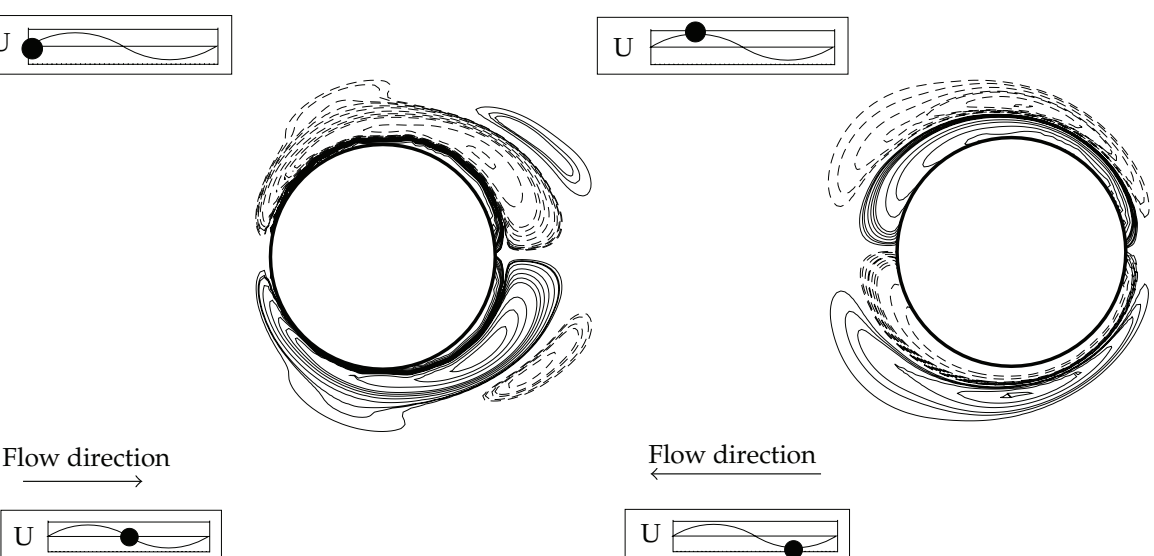

$\mathrm{U}$

Figure 2: Evolution of vorticity distribution at $K C=2$ and $R e=200$. Dash lines refer to the negative vorticity contours.

elongated as time increases. The predicted wake length in the model is very close to Iliadis and Anagnostopoulos' result. As KC increases, the symmetry in the pair of vortices breaks, Figure 4 shows the evolution of shedding vortices in a cycle. It is found that vortices are not attached to the cylinder anymore. They shed from the cylinder and travel downstream. Finally, they are damped in the far field region. In addition, the inline force coefficient $C_{f}$ is estimated using the proposed approach in the previous subsection. The time histories of $C_{f}$ at $\mathrm{KC}=2$ and 10 are shown in Figures 5(a) and 5(b), respectively. It is found that $C_{f}$ reaches a maximum while the oscillatory flow changes its direction. The comparison of the predicted $C_{f}$ reveals that the present immersed boundary model can simulate the interaction of oscillatory flow with a single cylinder and the hydrodynamic loading reasonably.

\subsection{Interaction of Oscillatory Flow with Cylinder Array}

A cylinder array is commonly used in marine structures. For example, a tension-leg platform which is normally used for the offshore production of oil or gas consists of a group of vertical cylinders as mentioned in Introduction. Those cylinders have to endure hydrodynamic loadings from waves and currents. To design those cylindrical structures, stress distribution caused by those hydrodynamic loadings must be provided in advance. Hence, the hydrodynamic loadings due to oscillatory flows are calculated using the current 


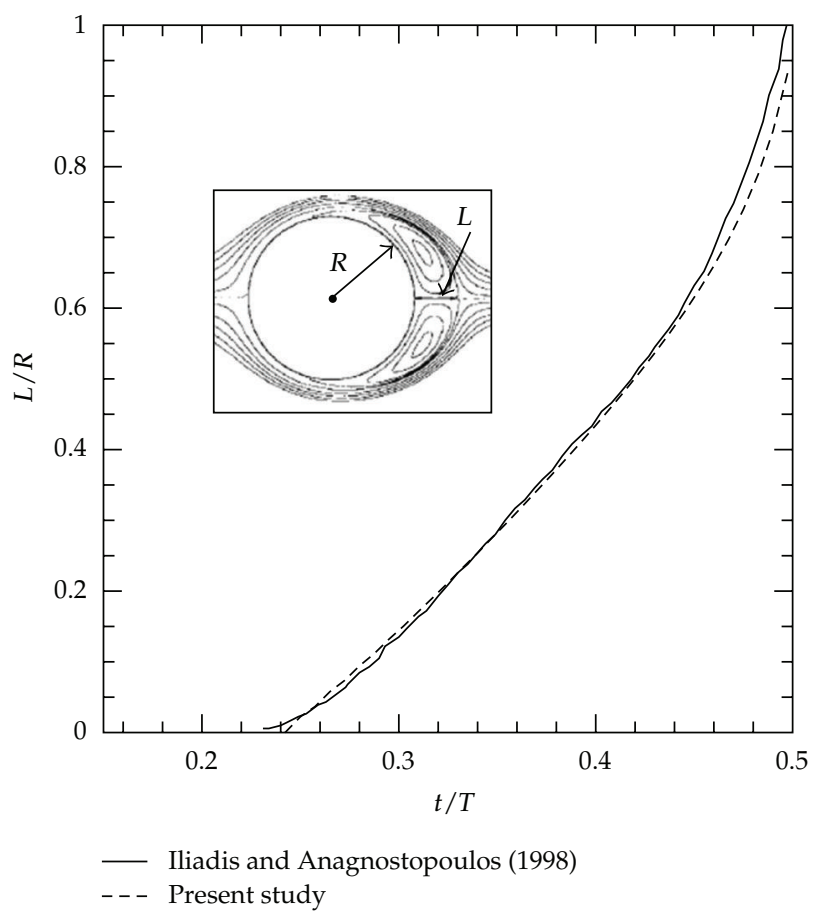

Figure 3: Evolution of wake during half a cycle at $\mathrm{KC}=2$ and $\operatorname{Re}=200$.

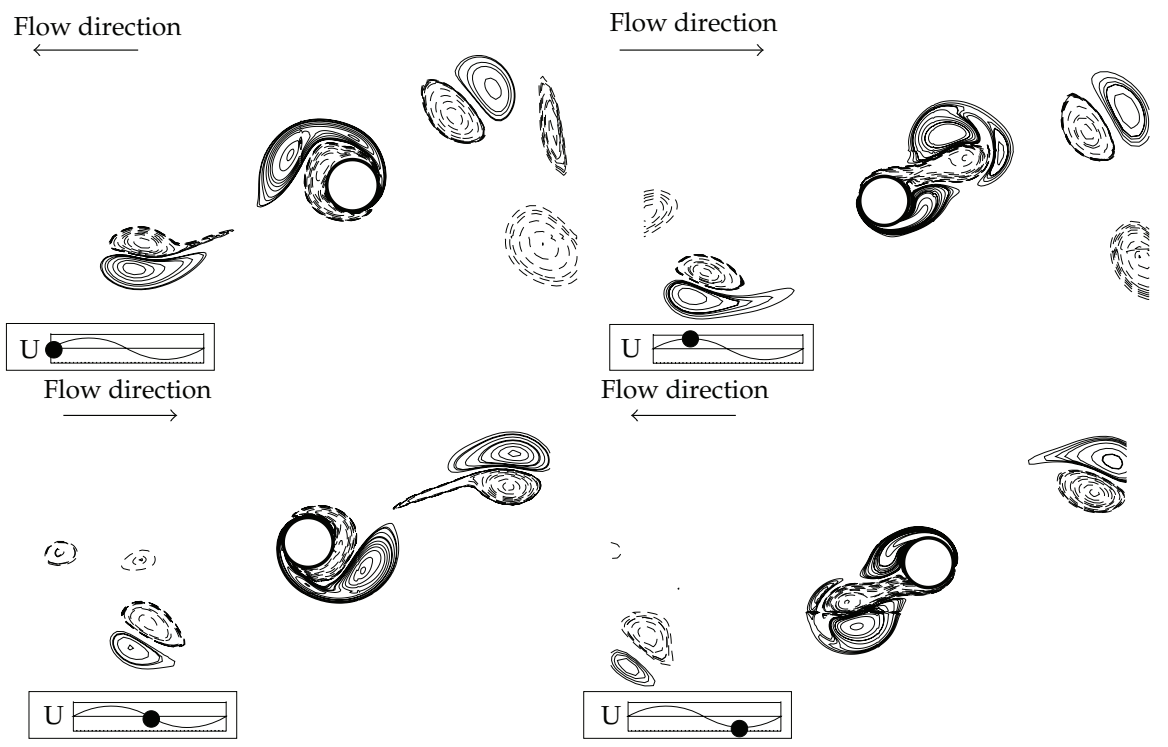

Figure 4: Evolution of vorticity distribution at $K C=10$ and $\operatorname{Re}=200$. Dash lines refer to the negative vorticity contours. 


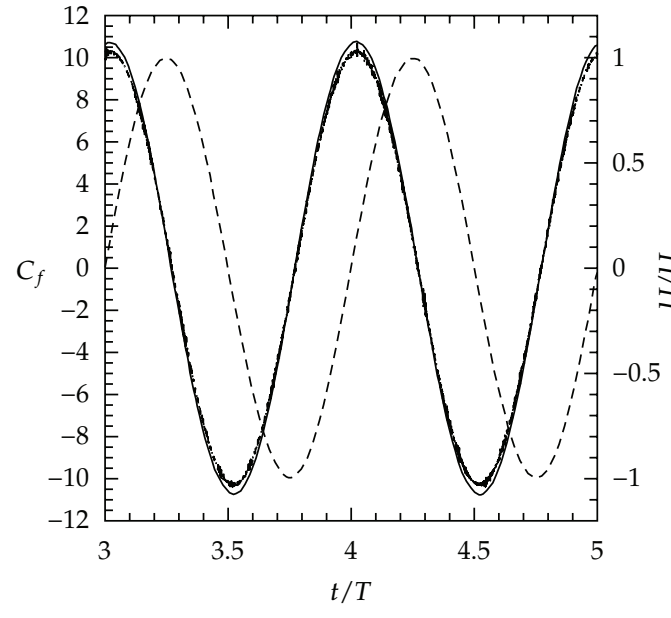

_ Iliadis and Anagnostopoulos (1998)
_. - Oscillatory flow velocity
... Present study

(a) $\mathrm{KC}=2$

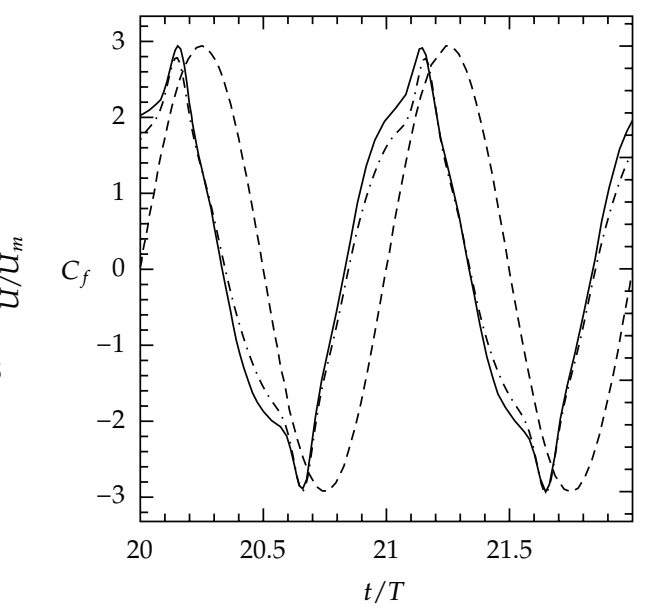

_ Iliadis and Anagnostopoulos (1998)

- - Oscillatory flow velocity

...- Present study

(b) $\mathrm{KC}=10$

Figure 5: Time histories of $C_{f}$ at (a) $K C=2$ and (b) $K C=10$.

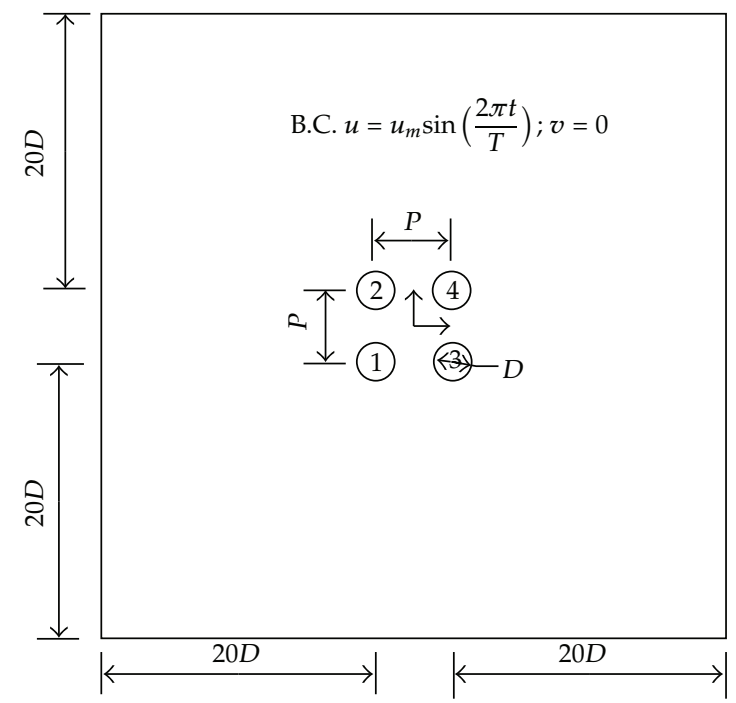

Figure 6: Schematic of interaction of an oscillatory flow with a cylinder array. $P / D=2$.

immersed boundary model. An array of four circular cylinders are considered in this study as shown in Figure 6. Four cylinders are allocated in a square arrangement. The distance between two centers of two adjacent cylinders is denoted as $P$. The pitch ratio $P / D$ is one of parameters to affect the hydrodynamic behavior around those cylinders. To simplify the problem, $P / D$ is set as 2 in this study. Also, Re is fixed at 200 in this subsection. To investigate the effect of KC number, three various KC numbers 2, 5, and 10 are considered to observe its effect on the oscillatory flow. Results are illustrated in the following subsections. 


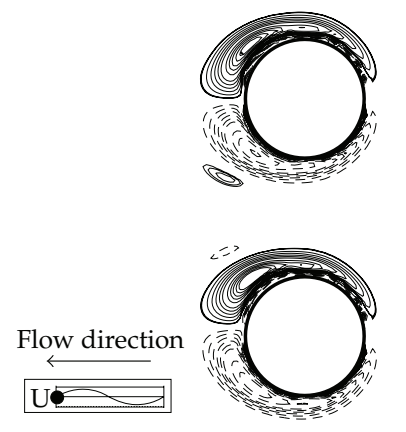

(a)
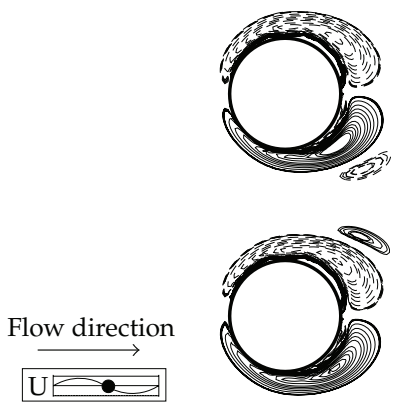

(c)
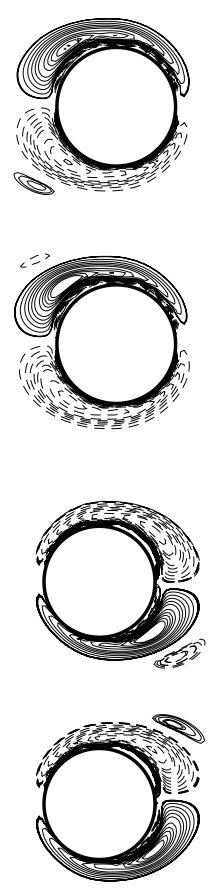

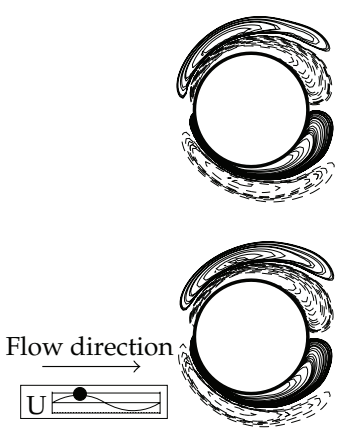

(b)
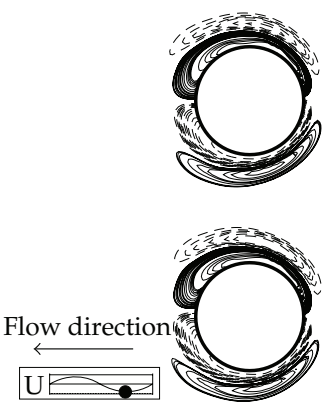

(d)
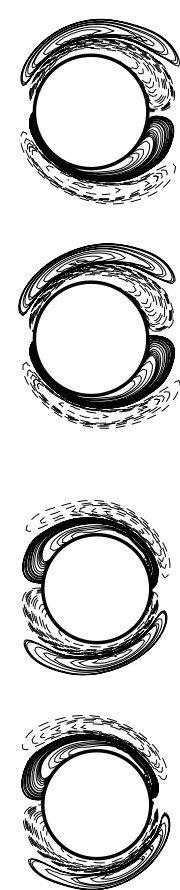

Figure 7: Snapshots of vorticity contours in the oscillatory flow interacting with four cylinders during a cycle at $\mathrm{KC}=2$ and $\operatorname{Re}=200$.

\subsubsection{Evolution of Four Vortical Systems}

Figure 7 shows the evolution of vorticity contours around four cylinders in a cycle. Each cylinder has its symmetric vortical system as mentioned in the case with a single cylinder. Those vortical systems do not interact with others since $P / D$ of 2 is wide. Nevertheless, the symmetry in those vortical systems vanishes as $\mathrm{KC}$ is increased to 5 . Figure 8 presents the snapshots of vorticity contours for the 11 th cycle at $\mathrm{KC}=5$. It is found that the gap flow influence the vortical systems, so vortices are pushed downstream. The vortices disappear after they leave from the cylinders. Figure 9 presents vorticity contours at $K C=10$. It is found that vortices become irregular and shed from cylinders. They are not damped until they travel far away from cylinders.

\subsubsection{Variation of $C_{f}$ with $K C$}

Prediction of hydrodynamic forces are important for the design of the cylinder array. To understand the variation of those forces with respect to $\mathrm{KC}$, the time histories of $C_{f}$ of the first cylinder as shown in Figure 6 are provided. Figure 10 demonstrates the time histories of $C_{f} . C_{f}$ behaves sinusoidaly at $K C=2$ as shown in Figure 10(a). It looks just like the case with a single cylinder. As $\mathrm{KC}$ increases to 5 , the sinusoidal form of $C_{f}$ is not regular any more as shown in Figure $10(\mathrm{~b})$. Also, the amplitude of $C_{f}$ decreases from 10 to 4 at $\mathrm{KC}=5$. As KC increases to $10, C_{f}$ becomes more irregular and also the amplitude decreases again. 


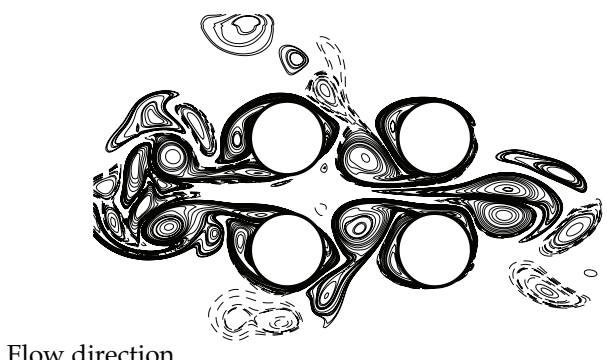

Flow direction

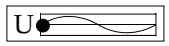

(a)

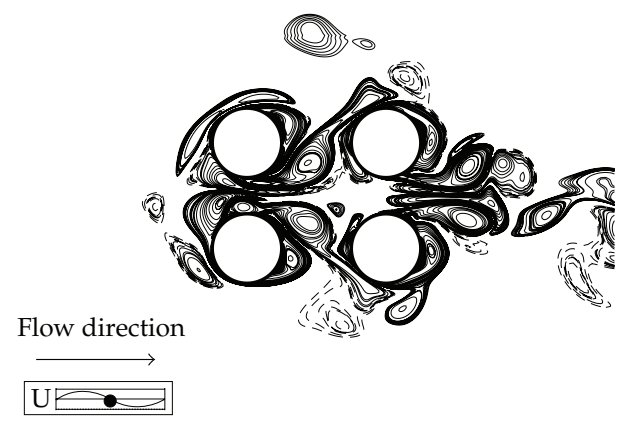

(c)

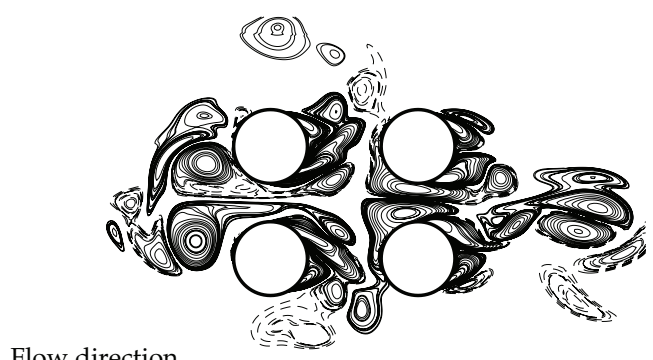

Flow direction

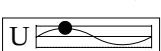

(b)

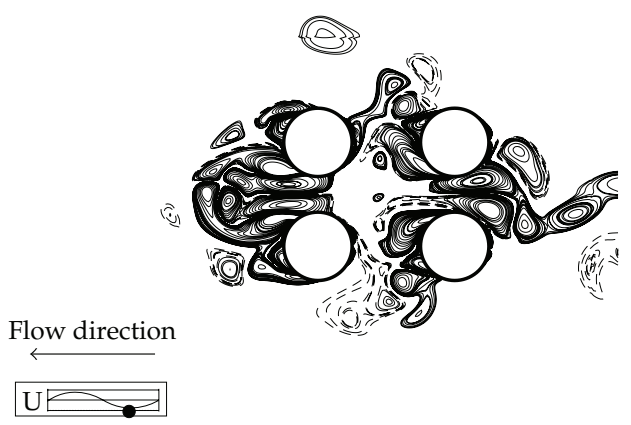

(d)

Figure 8: Snapshots of vorticity contours in the oscillatory flow interacting with four cylinders during a cycle at $\mathrm{KC}=5$ and $\operatorname{Re}=200$.

\subsubsection{Variation of $C_{l}$ with $K C$}

The other force component is along the transverse direction. It is called the life or transverse force. When $\mathrm{KC}=2$, as Figure 7 shows four vortical systems are symmetric during a cycle. As a result, lifts or transverse forces on four cylinders are very little in comparison with $C_{f}$. Nevertheless, as KC increases to 5, the vortical systems are not symmetric. The transverse forces on cylinders are obvious. Figure 11 shows time histories of $C_{l}$ at $K C=5$ and $10 . C_{l}$ at $\mathrm{KC}=5$ is not regular is smaller than $C_{f}$. While $\mathrm{KC}$ increases to 10 , the frequency of variation of $C_{l}$ becomes faster. Another feature at $\mathrm{KC}=10$ is those spikes. They do not appear regularly but randomly. This phenomenon does not occur in $C_{f}$.

\subsubsection{Phase Diagram of $C_{f}$ versus $C_{l}$}

The resultant hydrodynamic forces can be regarded as the responses of the vortical systems around those four cylinders. Trajectories of $C_{f}$ versus $C_{l}$ at successive instants reveal the states of the vortical systems. Figure 12 shows phase diagrams of $C_{f}$ and $C_{l}$ of the first cylinder at various $\mathrm{KC}$ numbers. While $\mathrm{KC}=2$, Figure 12(a) shows that the vortical system around the first cylinder is almost periodic. Therefore, the trajectory at $\mathrm{KC}=2$ is close to a straight line and the vortical system follows the line up and down. As KC increases to 5 and the transverse force becomes significant, the trajectory as shown in Figure 12(b) looks like a twisted " 8 ". Nevertheless, the trajectory shown in Figure 12(c) becomes completely chaotic 

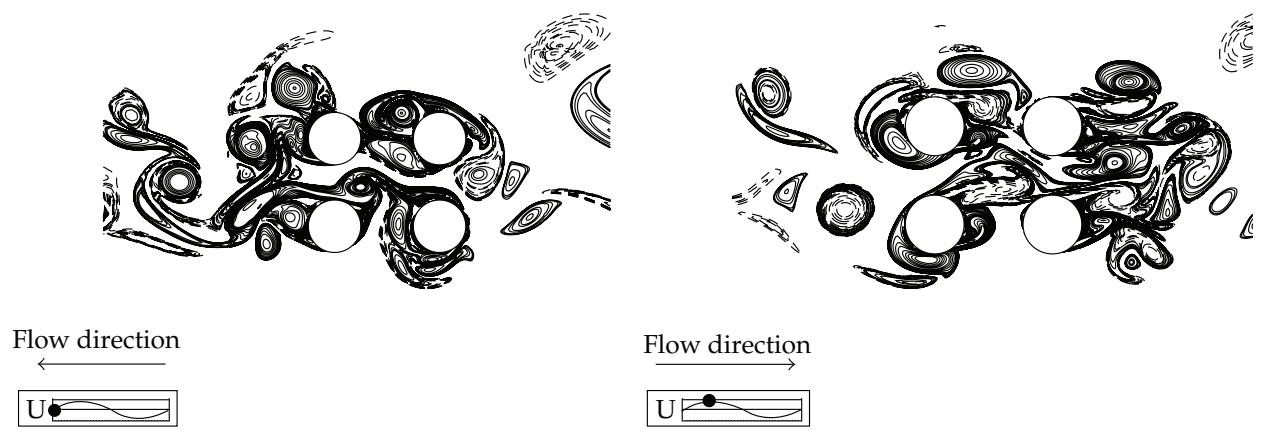

(a)

(b)

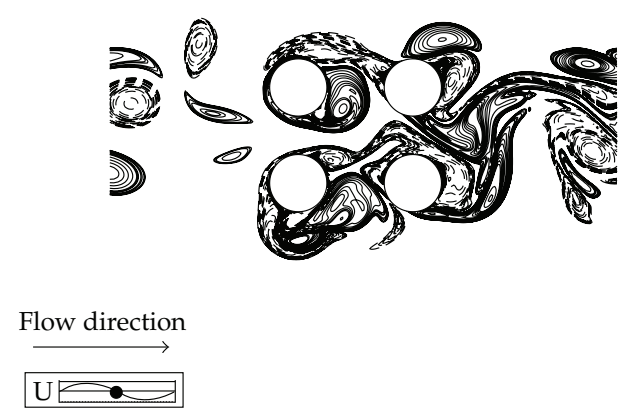

(c)

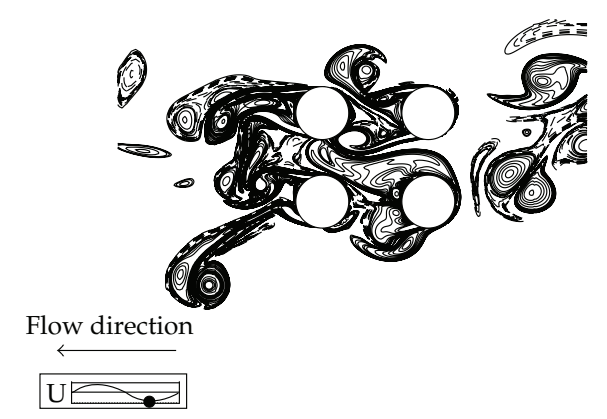

(d)

Figure 9: Snapshots of vorticity contours in the oscillatory flow interacting with four cylinders during a cycle at $\mathrm{KC}=10$ and $\mathrm{Re}=200$.

as $\mathrm{KC}$ increases to 10. In terms of those sub figures, it illustrates the way of the vortical system changes from a periodic state to a chaotic state due to the increase of $\mathrm{KC}$.

\section{Conclusions}

A direct forcing immersed boundary model has been established to predict interactions between oscillatory flows with a cylinder array. The proposed immersed boundary model was validated by an oscillating flow interacting with a single cylinder at Keulegan-Carpenter $(\mathrm{KC})$ number $=2$ and 10 and Reynolds number $=200$. The development of the symmetric wake predicted by the proposed model agreed with other researchers' study at $\mathrm{KC}=2$. Meanwhile, time histories of the inline force coefficient $C_{f}$ at $\mathrm{KC}=2$ and 10 were compared with the same published study. Good agreements were found between the present results and the other study. The established numerical model was further applied to simulate oscillatory flows around four cylinders at moderate KC numbers. As KC was 2, the symmetry was found in all vortical systems around four cylinders. The resultant $C_{f}$ of one of cylinders was sinusoidal and the lift coefficient $C_{l}$ was very small at $K C=2$. As $K C$ increased to 5 , the symmetry in vortical systems broke. $C_{f}$ became irregular and its amplitude decreased and $C_{l}$ was significant. While KC was 10 , the vortical systems were more complex. The change of $C_{f}$ became more frequent and a number of spikes were found in $C_{l}$. Phase diagrams of $C_{f}$ versus $C_{l}$ were used to demonstrate the state vortical system around the cylinder. It showed that the trajectory changed from a straight line to a twisted " 8 " as KC changed from 2 to 5. 


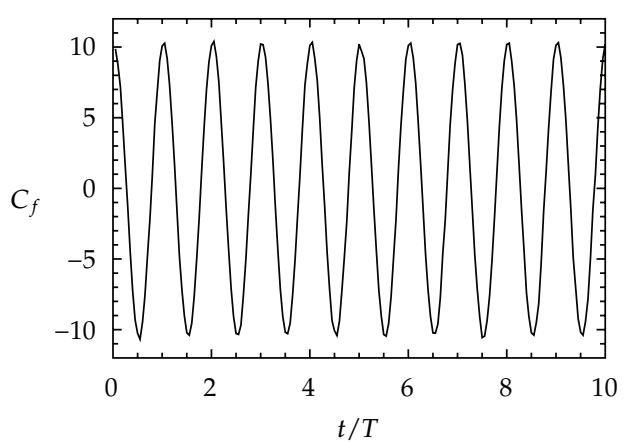

(a)

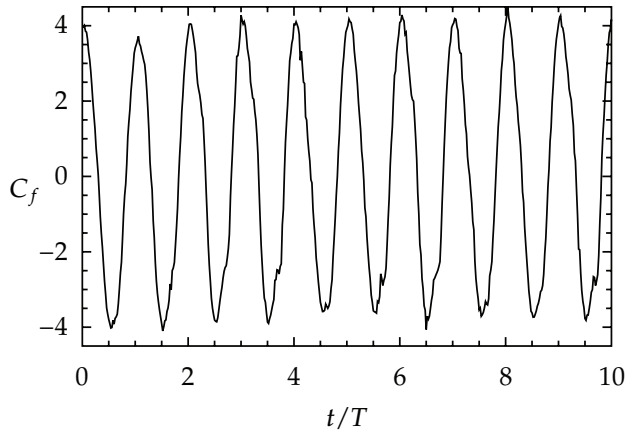

(b)

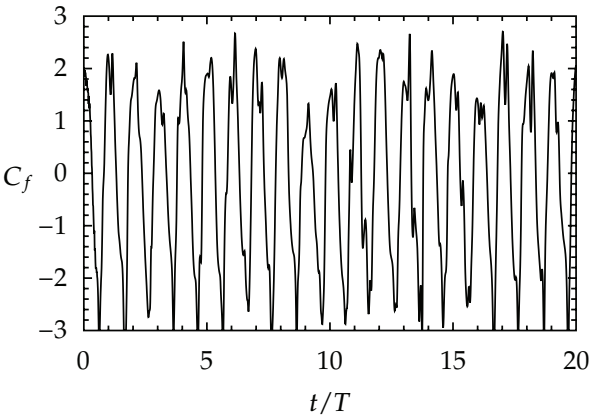

(c)

Figure 10: Time histories of $C_{f}$ of the first cylinder at $K C=2,5$, and 10.

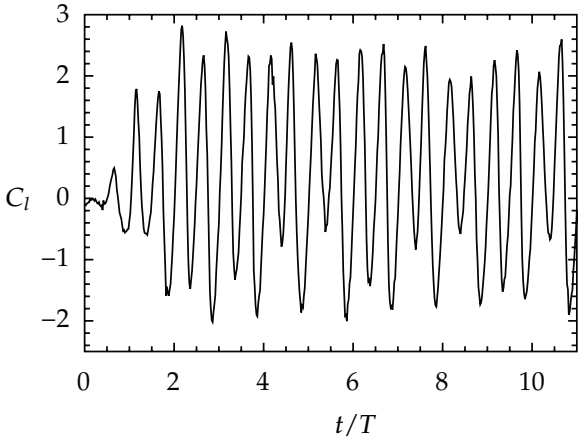

(a)

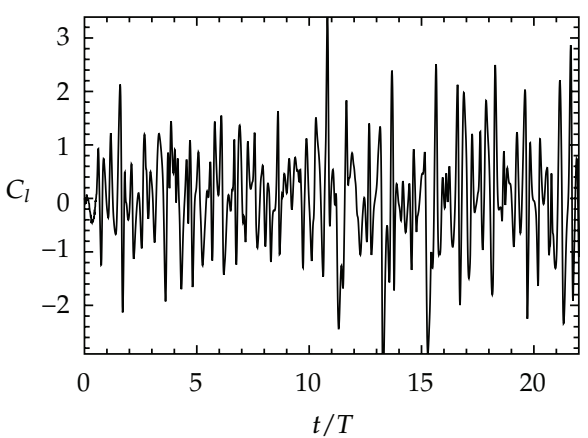

(b)

Figure 11: Time histories of $C_{l}$ of the first cylinder at $K C=5$ and 10.

Finally, when KC was 10, the trajectory in the phase diagram was completely chaotic. It meant that the vortical system around the cylinder was completely disordered. 


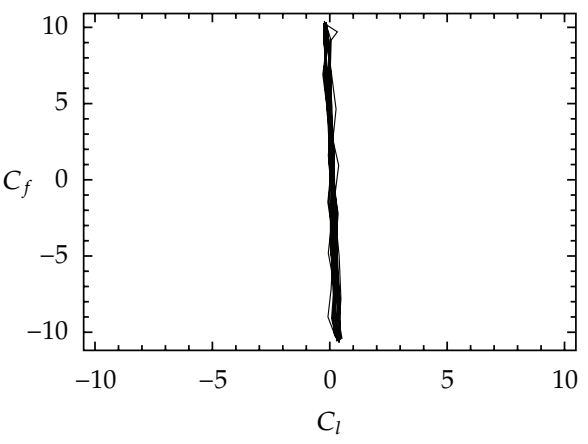

(a)

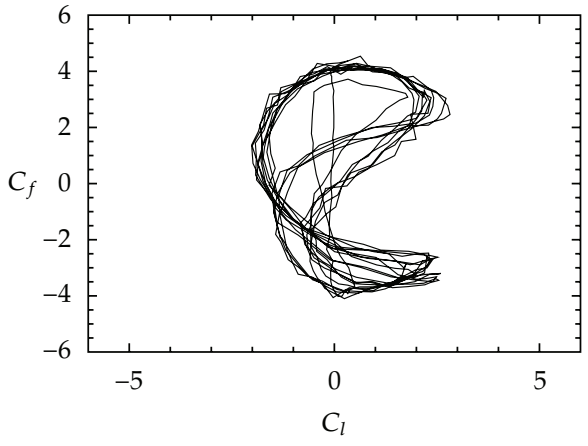

(b)

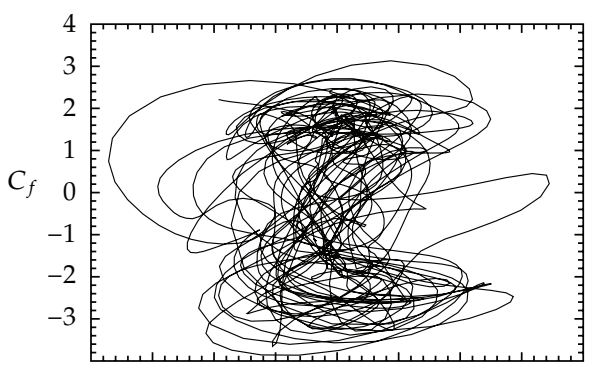

(c)

Figure 12: Phase diagrams of $C_{f}$ versus $C_{l}$ of the first cylinder at $K C=2,5$, and 10 .

\section{Nomenclatures}

\section{Latin Symbols}

$C_{f}: \quad$ Inline force coefficient

$C_{l}: \quad$ Lift coefficient

D: $\quad$ Diameter of cylinder

f: $\quad$ Dimensionless virtual force

F: $\quad$ Total dimensionless force acting on solids

KC: $\quad$ Keulegan-Carpenter number, $U_{m} T / D$

$P: \quad$ Dimensionless distance between centers of two adjacent cylinders

$L: \quad$ Dimensionless length of wake

$p: \quad$ Dimensionless pressure

$R: \quad$ Dimensionless radius of cylinder

Re: $\quad$ Reynolds number, $U_{m} D / v$

$T$ : $\quad$ Dimensionless period of oscillating velocity

$t$ : Dimensionless time

$\Delta t: \quad$ Dimensionless time increment

$U_{m}$ : Amplitude of oscillating velocity, $\mathrm{m} \cdot \mathrm{s}^{-1}$

$\mathbf{u}(u, v)$ : Dimensionless velocity

$x, y: \quad$ Dimensionless horizontal and vertical cartesian coordinates. 


\section{Greek Symbols}

$\eta$ : The volume of solid function

$v$ : Kinematic viscosity of fluid, $\mathrm{m}^{2} \cdot \mathrm{s}^{-1}$.

\section{Subscripts}

s: Solid.

\section{Superscripts}

$m$ : Time step level

*: Intermediate time step level.

\section{Acknowledgment}

The authors would like to express their gratitude for the financial support from National Science Council, Taiwan (Grant no.: NSC 100-2212-E-011-163).

\section{References}

[1] "Langlee Wave Power," http://www.langlee.no/.

[2] B. M. Sumer and J. Fredsoe, Hydrodynamics Around Cylindrical Structures, chapter 3, World Scientific Publishing, Singapore, 1997.

[3] C. H. K. Williamson, "Sinusoidal flow relative to circular cylinders," Journal of Fluid Mechanics, vol. 155, pp. 141-174, 1985.

[4] T. Sarpkaya, "Force on a circular cylinder in viscous oscillatory flow at low Keulegan-Carpenter numbers," Journal of Fluid Mechanics, vol. 165, pp. 61-71, 1986.

[5] E. D. Obasaju, P. W. Bearman, and J. M. R. Graham, "A study of forces, circulation and vortex patterns around a circular cylinder in oscillating flow," Journal of Fluid Mechanics, vol. 196, pp. 467-494, 1988.

[6] X. W. Lin, P. W. Bearman, and J. M. R. Graham, "A numerical study of oscillatory flow about a circular cylinder for low values of beta parameter," Journal of Fluids and Structures, vol. 10, no. 5, pp. 501-526, 1996.

[7] G. Iliadis and P. Anagnostopoulos, "Viscous oscillatory flow around a circular cylinder at low Keulegan-Carpenter numbers and frequency parameters," International Journal for Numerical Methods in Fluids, vol. 26, no. 4, pp. 403-442, 1998.

[8] W. Zheng and C. Dalton, "Numerical prediction of force on rectangular cylinders in oscillating viscous flow," Journal of Fluids and Structures, vol. 13, no. 2, pp. 225-249, 1999.

[9] H. An, L. Cheng, and M. Zhao, "Direct numerical simulation of oscillatory flow around a circular cylinder at low Keulegan-Carpenter number," Journal of Fluid Mechanics, vol. 666, pp. 77-103, 2011.

[10] M. J. Chern, P. Rajesh Kanna, Y. J. Lu, I. C. Cheng, and S. C. Chang, "A CFD study of the interaction of oscillatory flows with a pair of side-by-side cylinders," Journal of Fluids and Structures, vol. 26, no. 4, pp. 626-643, 2010.

[11] P. Anagnostopoulos and C. Dikarou, "Numerical simulation of viscous oscillatory flow past four cylinders in square arrangement," Journal of Fluids and Structures, vol. 27, no. 2, pp. 212-232, 2011.

[12] J. M. Yusof, Interaction of massive particles with turbulence [Ph.D. thesis], Department of Mechanical and Aerospace Engineering, Cornell University, Ithaca, NY, USA, 1996.

[13] E. A. Fadlun, R. Verzicco, P. Orlandi, and J. M. Yusof, "Combined immersed-boundary finitedifference methods for three-dimensional complex flow simulations," Journal of Computational Physics, vol. 161, no. 1, pp. 35-60, 2000.

[14] Y. H. Tseng and J. H. Ferziger, "A ghost-cell immersed boundary method for flow in complex geometry," Journal of Computational Physics, vol. 192, no. 2, pp. 593-623, 2003. 
[15] R. Verzicco, J. M. Yusof, P. Orlandi, and D. Haworth, “Large eddy simulation in complex geometric configurations using boundary body forces," American Institute of Aeronautics and Astronautics Journal, vol. 38, no. 3, pp. 427-433, 2000.

[16] D. Z. Noor, M. J. Chern, and T. L. Horng, "An immersed boundary method to solve fluid-solid interaction problems," Computational Mechanics, vol. 44, no. 4, pp. 447-453, 2009. 


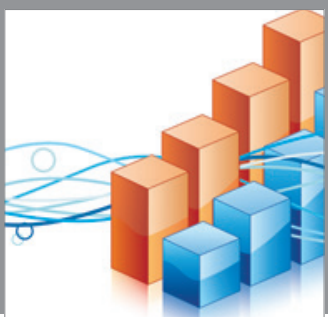

Advances in

Operations Research

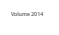

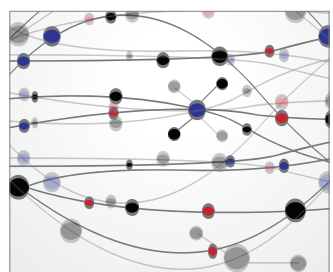

\section{The Scientific} World Journal
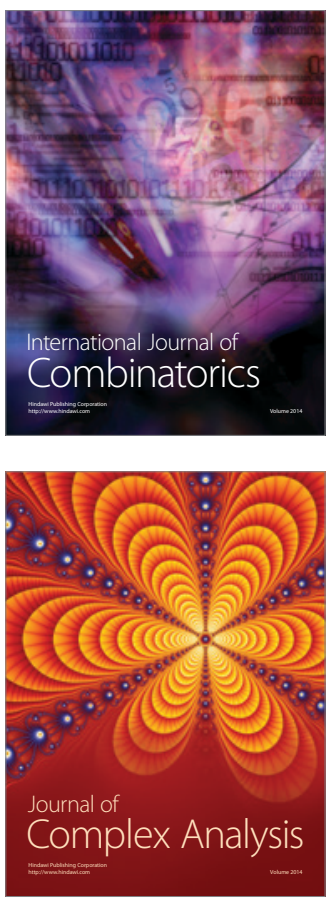

International Journal of

Mathematics and

Mathematical

Sciences
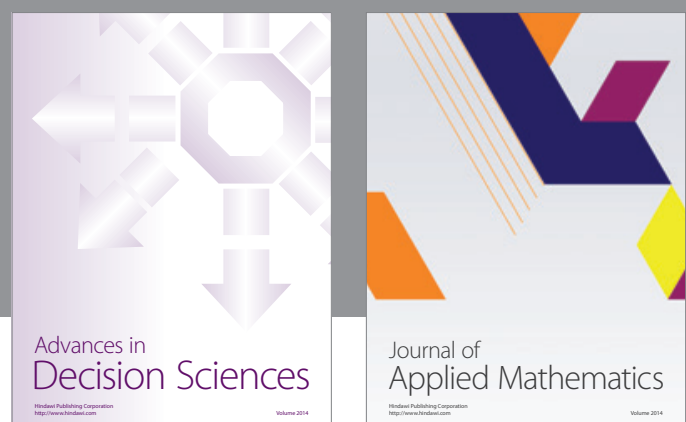

Journal of

Applied Mathematics
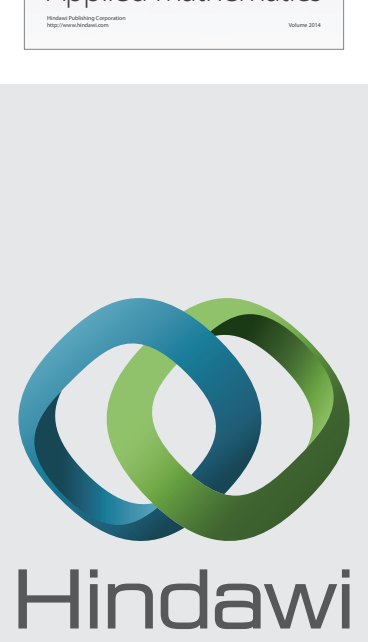

Submit your manuscripts at http://www.hindawi.com
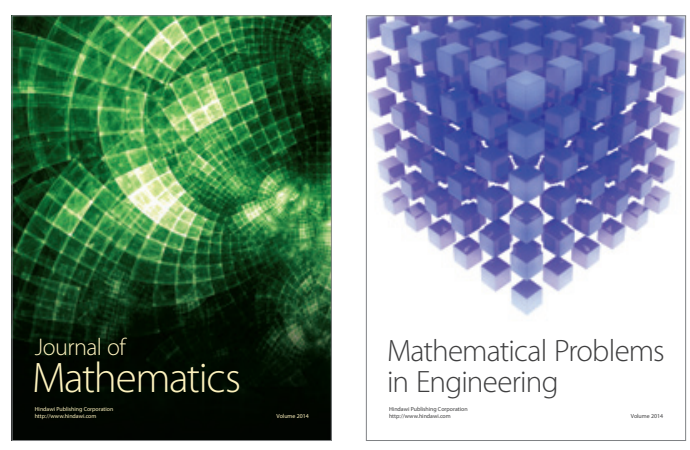

Mathematical Problems in Engineering
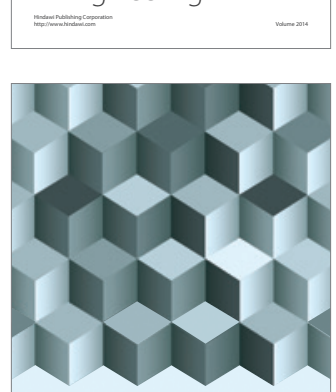

Journal of

Function Spaces
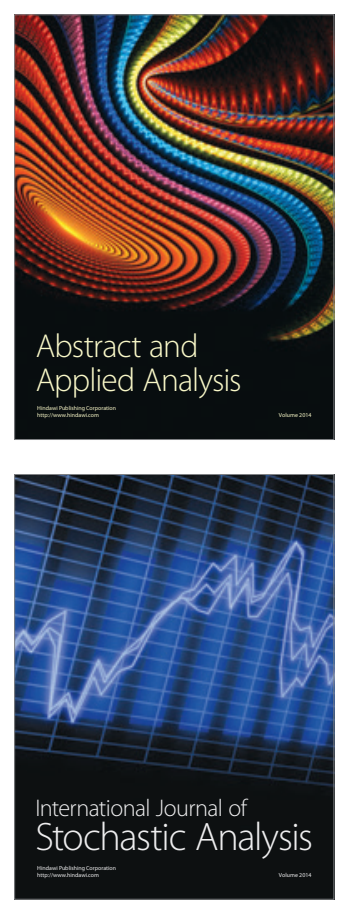

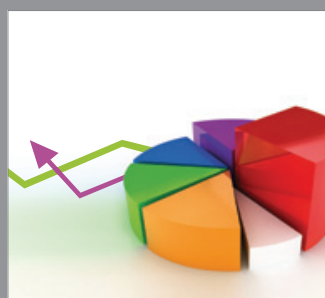

ournal of

Probability and Statistics

Promensencen
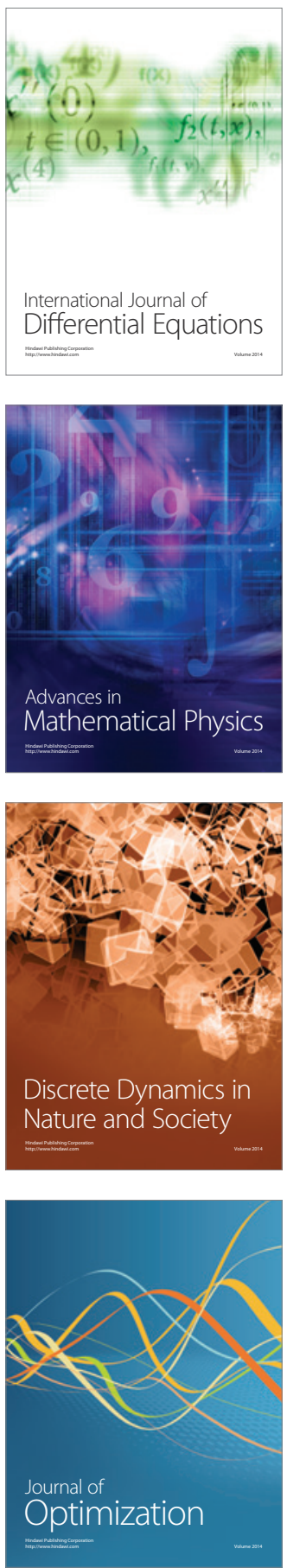